\title{
Electromechanical Behavior of PZT-Brass Unimorphs
}

\author{
Xiaoping Li, ${ }^{*},+$ Wan Y. Shih, $,{ }^{*},+\neq$ Ilhan A. Aksay, ${ }^{*}, \ddagger$ and Wei-Heng Shih ${ }^{*}, \dagger$ \\ Department of Materials Engineering, Drexel University, Philadelphia, Pennsylvania 19104-2875; and \\ Department of Chemical Engineering and Princeton Materials Institute, Princeton University, \\ Princeton, New Jersey 08544-5263
}

\begin{abstract}
We have examined the static and dynamic electromechanical responses of PZT-brass (piezoelectric-nonpiezoelectric) plate unimorphs of various brass/PZT thickness ratios. The study was performed both experimentally and theoretically. The static measurements showed that, given a PZT layer thickness, there exists a brass/PZT thickness ratio that gives the unimorph the highest static displacement under an applied field. The effects of geometric shape and external loading on the displacement of the unimorphs were also examined. The dynamic measurements showed that, given a PZT layer thickness, the bending-mode resonance frequencies increase with an increasing brass/PZT thickness ratio. These results were in good agreement with the theoretical predictions that were obtained with the plate geometry.
\end{abstract}

\section{Introduction}

$\mathrm{P}$ IEZOELECTRIC transducers can produce various modes of displacements. A flextensional-mode transducer generally produces a much higher displacement than an extensionalmode transducer. For example, a traditional piezoelectric bimorph, $4 \mathrm{~cm}$ long and $0.5 \mathrm{~mm}$ thick, can generate millimeterrange displacements, whereas an extensional-mode transducer produces only strains on the order of $10^{-4}$. Although a piezoelectric bimorph can generate large displacements, it has very limited loading capabilities. Recently, several new flextensional transducers have emerged. One is a metal-ceramic composite called the Moonie, ${ }^{1}$ which has a metal cap mounted on a piezoelectric disk, such as PZT (lead zirconate titanate), with a cavity between the central part of the metal cap and the PZT. The Rainbow (reduced and internally biased oxide wafer) ${ }^{2}$ is another transducer that is obtained by reducing one side of a piezoelectric disk, e.g., PLZT (lead lanthanum zirconate titanate). The reduced side is conductive enough to serve as an electrode. Detailed microstructure-property relationships of the Rainbow transducers have been recently investigated. ${ }^{3,4}$ The Moonie and the Rainbow show improved loading capabilities over the traditional bimorph. ${ }^{1,2}$ Other new flextensional transducers include Cerambow ${ }^{5}$ and Thunder. ${ }^{5,6}$ It has been claimed $^{6}$ that a Thunder actuator $2.54 \mathrm{~cm}$ in diameter and 0.58 $\mathrm{mm}$ in thickness can generate displacement close to $200 \mu \mathrm{m}$ under the electric field of $15 \mathrm{kV} / \mathrm{m}$.

The large displacements displayed by these new transducers are the results of gradients in the piezoelectric coefficient in the thickness direction. Although the design of the Moonie is different from that of the Rainbow, the underlying physics for the high displacements of both transducers is similar. Consider a

C. Randall—contributing editor

\footnotetext{
Manuscript No. 190432. Received January 22, 1998; approved November 20, 1998 *Member, American Ceramic Society.

Drexel University.

*Princeton University.
}

piezoelectric-nonpiezoelectric unimorph, as shown in Fig. 1. When a voltage is applied to the transducer, the gradient in the $d_{31}$ coefficient in the thickness direction generates a difference in the lateral strain between the two layers, causing the transducer to arch. A Rainbow transducer behaves in a similar fashion as in Fig. 1. The Moonie transducer behaves slightly differently in that the piezoelectric part does not bend; only the metal end cap arches up. Any transducer with a $d_{31}$ coefficient asymmetric with respect to the neutral axis would behave similarly. The simplest flextensional transducer with a gradient in $d_{31}$ would be a piezoelectric-nonpiezoelectric strip bimorph. ${ }^{7,8}$ The calculations of Shih et al. ${ }^{9}$ have shown that a piezoelectric-nonpiezoelectric strip transducer indeed can be used as a model to explain the various scaling behaviors exhibited by the displacements of Moonies and Rainbows. Besides the total thickness of the bimorph, the thickness ratio between the piezoelectric strip and the nonpiezoelectric strip can also affect the displacement. More recently, Li et al. ${ }^{10}$ have shown that the large displacement of Rainbow actuators can result from the stress-enhanced domain reorientation as well.

In addition to the large static axial displacement, the dynamic response of these $d_{31}$ gradient materials is also of great interest. ${ }^{4,11}$ Because the flextensional mode resonance vibration can be easily excited under alternating electric fields, these structures can be used as components for sensing applications over a broad range of frequency. The resonance frequency is related to the geometric shape as well as the elastic and piezoelectric properties of the materials. A better understanding of the resonance behavior of unimorphs is essential to better design the sensors.

In this article, we present experimental and theoretical results on the static displacements as well as the dynamic response of piezoelectric-nonpiezoelectric unimorphs with various thickness ratios and shapes. Unlike the previous study, ${ }^{9}$ the present calculations have been performed with plate geometry to account for the shape effect. The piezoelectric-nonpiezoelectric system studied is the PZT-brass unimorph. The static

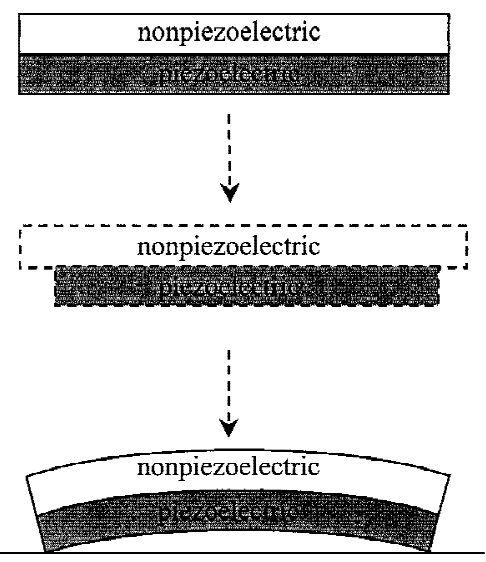

Without electric field Stress free

With electric field Unconstrained

Fig. 1. Schematic behavior of a nonpiezoelectric-piezoelectric unimorph under an electric field. 
results show that the PZT-brass unimorph can achieve similarly high displacements as the Rainbow transducers. Furthermore, for a given PZT layer thickness, there exists an optimal brass/PZT thickness ratio that produces the highest displacement. The results of dynamic measurements show that the bending-mode resonance frequency is very sensitive to the brass/PZT thickness ratio. For a given PZT thickness, the bending-mode resonance frequencies increase with increasing brass/ PZT thickness ratio. Both the results of the static and dynamic measurements are in good agreement with the theoretical predictions.

\section{Theory}

In this article, the previous theory that we have developed for the displacement of nonpiezoelectric-piezoelectric strips is modified to describe plates rather than beams. We consider a piezoelectric-nonpiezoelectric unimorph with a piezoelectric layer of thickness $t_{\mathrm{p}}$ and Young's modulus $E_{\mathrm{p}}$ and a nonpiezoelectric layer of thickness $t_{\mathrm{np}}$ and Young's modulus $E_{\mathrm{np}}$, as schematically shown in Fig. 2. The bonding layer between the piezoelectric and nonpiezoelectric layers is neglected in this theory. A perfect bonding between the two layers is assumed. When an electric field $E$ is applied in the $\mathbf{z}$ (thickness) direction, a lateral strain $d_{31} E$ is generated in the piezoelectric layer, where $d_{31}$ is the piezoelectric coefficient of the piezoelectric layer, causing the unimorph to bend. The position of a neutral plane, $t_{\mathrm{n}}$, is determined by

$$
\int_{-t_{\mathrm{np}}}^{0} E_{\mathrm{np}}\left(\frac{z-t_{\mathrm{n}}}{r}\right) \mathrm{d} z+\int_{0}^{t_{\mathrm{p}}} E_{\mathrm{p}}\left(\frac{z-t_{\mathrm{n}}}{r}\right) \mathrm{d} z=0
$$

where $r$ is the radius of curvature due to bending. Once the position of the neutral plane is known, the bending modulus per unit length, $D$, of the piezoelectric-nonpiezoelectric unimorph can be calculated, according to

$$
D=\int_{-t_{\mathrm{np}}}^{0} E_{\mathrm{np}}\left(z-t_{\mathrm{n}}\right)^{2} \mathrm{~d} z+\int_{0}^{t_{\mathrm{p}}} E_{\mathrm{p}}\left(z-t_{\mathrm{n}}\right)^{2} \mathrm{~d} z
$$

yielding

$$
D=\frac{E_{\mathrm{p}}^{2} t_{\mathrm{p}}^{4}+E_{\mathrm{np}}^{2} t_{\mathrm{np}}^{4}+2 E_{\mathrm{p}} E_{\mathrm{np}} t_{\mathrm{p}} t_{\mathrm{np}}\left(2 t_{\mathrm{p}}^{2}+2 t_{\mathrm{np}}^{2}+3 t_{\mathrm{p}} t_{\mathrm{np}}\right)}{12\left(E_{\mathrm{p}} t_{\mathrm{p}}+E_{\mathrm{np}} t_{\mathrm{np}}\right)}
$$

The bending moments per unit length in the $\mathbf{x}$ and $\mathbf{y}$ directions $M_{\mathbf{x}, \mathrm{p}}$ and $M_{\mathbf{y}, \mathrm{p}}$, resulting from the piezoelectic layer, and $M_{\mathbf{x}, \mathrm{np}}$ and $M_{\mathbf{y}, \mathrm{np}}$, resulting from the nonpiezoelectric layer, can be calculated according to

$$
\begin{aligned}
M_{\mathbf{x}, \mathrm{p}}=M_{\mathbf{y}, \mathrm{p}} & =\int_{0}^{t_{\mathrm{p}}} E_{\mathrm{p}}\left(d_{31} E-c\right)\left(z-t_{\mathrm{n}}\right) \mathrm{d} z \\
& =\frac{E_{\mathrm{np}} t_{\mathrm{np}}}{\left(E_{\mathrm{np}} t_{\mathrm{np}}+E_{\mathrm{p}} t_{\mathrm{p}}\right)} M_{\mathrm{E}} \\
M_{\mathbf{x}, \mathrm{np}}=M_{\mathbf{y}, \mathrm{np}} & =\int_{-t_{\mathrm{np}}}^{0} E_{\mathrm{np}}(-c)\left(z-t_{\mathrm{n}}\right) \mathrm{d} z \\
& =\frac{E_{\mathrm{p}} t_{\mathrm{p}}}{\left(E_{\mathrm{np}} t_{\mathrm{np}}+E_{\mathrm{p}} t_{\mathrm{p}}\right)} M_{\mathrm{E}}
\end{aligned}
$$

where

$$
M_{\mathrm{E}}=\frac{E_{\mathrm{p}} E_{\mathrm{np}} t_{\mathrm{p}} t_{\mathrm{np}}\left(t_{\mathrm{p}}+t_{\mathrm{np}}\right) d_{31} E}{2\left(E_{\mathrm{np}} t_{\mathrm{np}}+E_{\mathrm{p}} t_{\mathrm{p}}\right)}
$$

and $c$ is the in-plane constrain that satisfies

$$
\int_{-t_{\mathrm{np}}}^{0} E_{\mathrm{np}} c \mathrm{~d} z+\int_{0}^{t_{\mathrm{p}}} E_{\mathrm{p}}\left(c-d_{31} E\right) \mathrm{d} z=0
$$

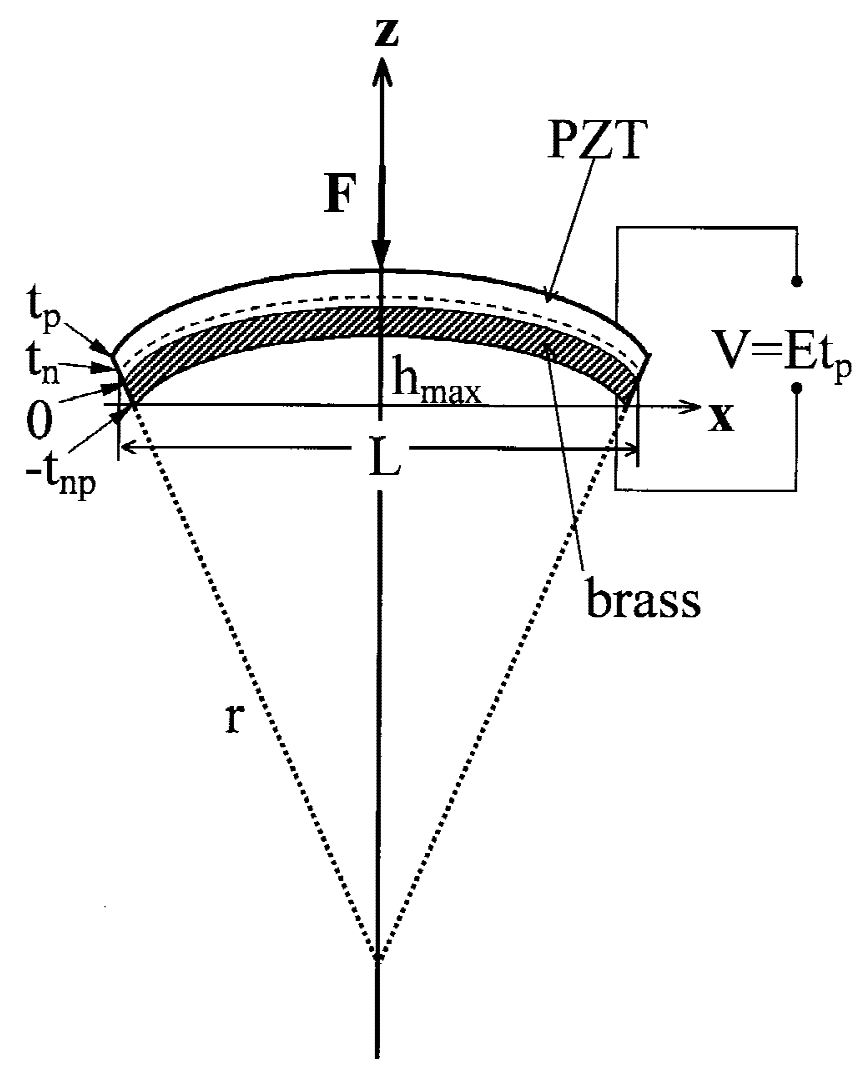

Fig. 2. Schematic and coordinates of a unimorph.

\section{(1) Displacements of Rectangular and Square Unimorphs}

For a rectangular unimorph of edges $L_{\mathbf{x}}$ and $L_{\mathbf{y}}$ lying in the $\mathbf{x}$ and $\mathbf{y}$ plane, the flexural displacement $h$ in the $\mathbf{z}$ direction obeys the following differential equation:

$$
\left(\frac{\partial^{2}}{\partial x^{2}}+\frac{\partial^{2}}{\partial y^{2}}\right) h=-\frac{M^{\prime}}{D}
$$

where $M^{\prime}$ can be calculated as

$$
M^{\prime}=\left(1-v_{\mathrm{p}}\right)\left(M_{\mathbf{x}, \mathrm{p}}+M_{\mathbf{y}, \mathrm{p}}\right)+\left(1+v_{\mathrm{np}}\right)\left(M_{\mathbf{x}, \mathrm{np}}+M_{\mathbf{y}, \mathrm{np}}\right)
$$

where $v_{\mathrm{p}}$ and $v_{\mathrm{np}}$ are the Poisson ratios of the piezoelectric and nonpiezoelectric layers, respectively. Assuming $\nu_{\mathrm{p}}=v_{\mathrm{np}}=\nu$,

$$
M^{\prime}=(1-v)\left[\left(M_{\mathbf{x}, \mathrm{p}}+M_{\mathbf{x}, \mathrm{np}}\right)+\left(M_{\mathbf{y}, \mathrm{p}}+M_{\mathbf{y}, \mathrm{np}}\right)\right]
$$

The solution to Eq. (8) can be obtained as

$$
h=\frac{1}{4} \frac{M^{\prime}}{D}\left[x\left(L_{\mathbf{x}}-x\right)+y\left(L_{\mathbf{y}}-y\right)\right]
$$

when the plate is simply supported at its four corners. For a rectangular plate, the maximum displacement occurs at the center of the plate:

$$
h_{\text {max }, \text { rect }}=\frac{1}{16} \frac{M^{\prime}}{D}\left(L_{\mathbf{x}}^{2}+L_{\mathbf{y}}^{2}\right)
$$

For a square plate, $L_{\mathbf{x}}=L_{\mathbf{y}}=L$, and

$$
\begin{aligned}
h_{\mathrm{max}, \mathrm{sq}} & =\frac{1}{8} \frac{M^{\prime}}{D} L^{2} \\
& =\frac{L^{2}}{4} \frac{6 E_{\mathrm{p}} E_{\mathrm{np}} t_{\mathrm{p}} t_{\mathrm{np}}\left(t_{\mathrm{p}}+t_{\mathrm{np}}\right) d_{31} E(1-v)}{E_{\mathrm{p}}^{2} t_{\mathrm{p}}^{4}+E_{\mathrm{np}}^{2} t_{\mathrm{np}}^{4}+2 E_{\mathrm{p}} E_{\mathrm{np}} t_{\mathrm{p}} t_{\mathrm{np}}\left(2 t_{\mathrm{p}}^{2}+2 t_{\mathrm{np}}^{2}+3 t_{\mathrm{p}} t_{\mathrm{np}}\right)}
\end{aligned}
$$


For a beam of length $L$ in the $\mathbf{x}$ direction and $L_{\mathbf{y}} \approx 0, h_{\text {max,beam }} \approx$ $0.5 h_{\text {max }, \mathrm{sq}}$.

\section{(2) Displacements of Circular Unimorphs}

For circular unimorphs, the displacement in the $\mathbf{z}$ direction obeys the following differential equation:

$$
\left(\frac{\partial^{2}}{\partial r^{2}}+\frac{1}{r} \frac{\partial}{\partial r}+\frac{1}{r^{2}} \frac{\partial^{2}}{\partial \theta^{2}}\right) h=-\frac{M^{\prime}}{D}
$$

where $D$ is the bending modulus per unit length as defined in Eq. (3) and $M^{\prime}$ the bending modulus as defined in Eqs. (4)-(6) and (9). The solution of Eq. (13) is

$$
h=\frac{M^{\prime}}{4 D}\left(R^{2}-r^{2}\right)
$$

when the circular unimorph is simply supported at the rim. The maximum displacement $h_{\max }$ occurs at the center and is given by the following expression:

$$
\begin{aligned}
h_{\text {max }, \mathrm{cir}} & =\frac{M^{\prime}}{4 D} R^{2}=\frac{M^{\prime}}{16 D} L^{2} \\
& =\frac{L^{2}}{8} \frac{6 E_{\mathrm{p}} E_{\mathrm{np}} t_{\mathrm{p}} t_{\mathrm{np}}\left(t_{\mathrm{p}}+t_{\mathrm{np}}\right) d_{31} E(1-v)}{E_{\mathrm{p}}^{2} t_{\mathrm{p}}^{4}+E_{\mathrm{np}}^{2} t_{\mathrm{np}}^{4}+2 E_{\mathrm{p}} E_{\mathrm{np}} t_{\mathrm{p}} t_{\mathrm{np}}\left(2 t_{\mathrm{p}}^{2}+2 t_{\mathrm{np}}^{2}+3 t_{\mathrm{p}} t_{\mathrm{np}}\right)}
\end{aligned}
$$

Comparing Eqs. (12) and (15) shows that, for a unimorph of width $L$, the displacement would be twice as much when it adapts to a square shape of edge $L$ as that when it adapts to a circular shape of diameter $L$.

Considering circular and square unimorphs with the same area $A, L^{2}=A$ for the square unimorph, whereas $L^{2}=4 A / \pi$ for the circular unimorph. Substituting the $L^{2}$ values in Eqs. (12) and (15) shows that the displacement of a square unimorph is $\pi / 2$ times that of a circular unimorph with the same area.

\section{(3) Loading Effect}

The effect of loading is illustrated by considering a beam of length $L_{\mathbf{x}}=L$ and width $L_{\mathbf{y}} \ll L$ with a concentrated load $F$ at the center and an electric field $E$ applied across the thickness, as shown in Fig. 1. The differential equation for the displacement $h$ can be expressed as

$$
\begin{aligned}
& \frac{\mathrm{d}^{2}}{\mathrm{~d} x^{2}} h=-\frac{M^{\prime}}{2 D}+\frac{F x}{2 D} \quad \text { for } x \leq L / 2 \\
& \frac{\mathrm{d}^{2}}{\mathrm{~d} x^{2}} h=-\frac{M^{\prime}}{2 D}+\frac{F x}{2 D}-\frac{F}{D}\left(x-\frac{L}{2}\right) \quad \text { for } L / 2<x<L
\end{aligned}
$$

The solution for Eq. (16) is

$$
\begin{aligned}
h=- & \frac{1}{4} \frac{M^{\prime}}{D}\left[x\left(x-L_{\mathbf{x}}\right)+y\left(y-L_{\mathbf{y}}\right)\right] \\
& -\frac{F x}{12 D}\left(\frac{3}{4} L^{2}-x^{2}\right) \quad \text { for } 0<x<L / 2 \\
h= & -\frac{1}{4} \frac{M^{\prime}}{D}\left[x\left(x-L_{\mathbf{x}}\right)+y\left(y-L_{\mathbf{y}}\right)\right]-\frac{F x}{12 D}\left(\frac{3}{4} L^{2}-x^{2}\right) \\
& -\frac{F[x-(L / 2)]^{3}}{6 D} \quad \text { for } L / 2<x<L
\end{aligned}
$$

when the beam is simply supported at the two ends. The maximum displacement occurs at $x=L / 2$ :

$$
\begin{aligned}
h_{\mathrm{max}} & =\frac{M^{\prime} L^{2}}{16 D}-\frac{F L^{3}}{48 D} \\
& =\frac{L^{2}}{8} \frac{6 E_{\mathrm{p}} E_{\mathrm{np}} t_{\mathrm{p}} t_{\mathrm{np}}\left(t_{\mathrm{p}}+t_{\mathrm{np}}\right) d_{31} E(1-v)-2\left(E_{\mathrm{p}} t_{\mathrm{p}}+E_{\mathrm{np}} t_{\mathrm{np}}\right) F L}{E_{\mathrm{p}}^{2} t_{\mathrm{p}}^{4}+E_{\mathrm{np}}^{2} t_{\mathrm{np}}^{4}+2 E_{\mathrm{p}} E_{\mathrm{np}} t_{\mathrm{p}} t_{\mathrm{np}}\left(2 t_{\mathrm{p}}^{2}+2 t_{\mathrm{np}}^{2}+3 t_{\mathrm{p}} t_{\mathrm{np}}\right)}
\end{aligned}
$$

\section{(4) Bending-Mode Resonance Frequency}

The bending-mode vibration of the unimorph obeys the following differential equation:

$$
D^{\prime} \nabla^{2} \nabla^{2} h=-m \frac{\partial^{2} h}{\partial t^{2}}
$$

where

$D^{\prime}=\frac{1}{1-v_{\mathrm{np}}^{2}} \int_{-t_{\mathrm{np}}}^{0} E_{\mathrm{np}}\left(z-t_{\mathrm{n}}\right)^{2} \mathrm{~d} z+\frac{1}{1-v_{\mathrm{p}}^{2}} \int_{0}^{t_{\mathrm{p}}} E_{\mathrm{p}}\left(z-t_{\mathrm{n}}\right)^{2} \mathrm{~d} z$

and

$$
m=\rho_{\mathrm{p}} t_{\mathrm{p}}+\rho_{\mathrm{np}} t_{\mathrm{np}}
$$

In Eqs. (20) and (21), $\rho_{\mathrm{p}}$ and $\rho_{\mathrm{np}}$ are the densities of the piezoelectric and nonpiezoelectric layers, respectively. In general, the resonance frequency of a plate obeys the following form:

$$
f=\frac{1}{2 \pi}\left(\frac{\lambda}{L}\right)^{2} \sqrt{\frac{D^{\prime}}{m}}
$$

where $\lambda$ is the eigenvalue of Eq. (19), is dimensionless, and depends only on the shape of the plate and the boundary conditions. If we consider $\nu_{\mathrm{p}}=v_{\mathrm{np}}=v$ and $D^{\prime}=D /\left(1-v^{2}\right)$, then we obtain

$f=\frac{1}{2 \pi}\left(\frac{\lambda}{L}\right)^{2} \sqrt{\frac{E_{\mathrm{p}}^{2} t_{\mathrm{p}}^{4}+E_{\mathrm{np}}^{2} t_{\mathrm{np}}^{4}+2 E_{\mathrm{p}} E_{\mathrm{np}} t_{\mathrm{p}} t_{\mathrm{np}}\left(2 t_{\mathrm{p}}^{2}+2 t_{\mathrm{np}}^{2}+3 t_{\mathrm{p}} t_{\mathrm{np}}\right)}{12\left(E_{\mathrm{p}} t_{\mathrm{p}}+E_{\mathrm{np}} t_{\mathrm{np}}\right)\left(\rho_{\mathrm{p}} t_{\mathrm{p}}+\rho_{\mathrm{np}} t_{\mathrm{np}}\right)\left(1-v^{2}\right)}}$

For the free-standing boundary conditions that were adopted in the experiment, the eigenvalues of the first two bending modes are $\lambda_{1}=6.028$ and $\lambda_{2}=12.42$ for disks and $\lambda_{1}=4.93$ and $\lambda_{2}=7.98$ for square plates. ${ }^{12}$

\section{Experimental Procedure}

The present piezoelectric-nonpiezoelectric unimorph transducers were made of PZT $\left(\mathrm{PbO}-\mathrm{ZrO}_{2}-\mathrm{TiO}_{2}\right)$ and brass. The shapes of the transducers studied included squares, rectangles, and disks. The piezoelectric PZT (APC PZT-857, APC International, Ltd., Mackeyville, PA) had silver paste electrodes on both sides. The brass layers used were brass sheets (Alloy 260, McMaster-Carr Supply Co., Dayton, NJ). The dimensions of each transducer and the relevant constants of both the PZT and the brass are listed in Table I. The materials characteristics of PZT and brass were taken from the data sheets provided by the suppliers. The poling was done in transformer oil with a temperature of $100^{\circ} \mathrm{C}$ and an electrical field of $2000 \mathrm{~V} / \mathrm{mm}$.

The surfaces of the PZT and the brass were cleaned before the PZT was glued to the brass with a conductive epoxy (GC Electronics, Rockford, IL). The volume resistivity of the conductive epoxy was $<5 \times 10^{-3} \Omega \cdot \mathrm{cm}$. After the PZT was glued to the brass, the unimorph was uniformly pressed at $\sim 0.03 \mathrm{MPa}$ for one day for curing. The thickness of the epoxy layer was $\sim 10 \mu \mathrm{m}$ for all unimorphs.

The static displacements of the unimorphs were measured with a linear-variable differential transformer (LVDT) (Model L500, Omega Engineering, Inc., Stamford, CT) with a resolution of $1 \mu \mathrm{m}$. In the measurement, square waveform electric fields were applied to the sample. A voltage $V$ was applied in unipolar waveform at $0.05 \mathrm{~Hz}$. The displacement under the applied voltage was recorded after three periods. The displacements of the unimorphs were measured with and without a load. The experimental setups with and without a load are 
Table I. Dimensions and Constants of Transducers Used in This Study

\begin{tabular}{|c|c|c|c|c|c|c|c|c|c|c|c|c|c|}
\hline & \multicolumn{13}{|c|}{ Sample $^{\dagger}$} \\
\hline & D1 & D2 & D3 & D4 & D5 & S1 & S2 & S3 & S4 & R1 & R2 & R3 & $\mathrm{R} 4$ \\
\hline $\begin{array}{l}\text { Diameter } \\
\text { or length } \\
(\mathrm{cm}) \\
\text { PZT }\end{array}$ & 2.54 & 2.54 & 2.54 & 2.54 & 3.12 & $\begin{array}{r}1.52 \times \\
1.52\end{array}$ & $\begin{array}{r}1.52 \times \\
1.52\end{array}$ & $\begin{array}{r}1.52 \times \\
1.52\end{array}$ & $\begin{array}{r}1.52 \times \\
1.52\end{array}$ & $\begin{array}{c}2.03 \times \\
0.254\end{array}$ & $\begin{array}{c}2.03 \times \\
0.508\end{array}$ & $\begin{array}{c}2.03 \times \\
0.813\end{array}$ & $\begin{array}{c}2.03 \times \\
1.016\end{array}$ \\
\hline $\begin{array}{l}\text { thickness } \\
(\mathrm{cm}) \\
\text { Brass }\end{array}$ & 0.1 & 0.1 & 0.1 & 0.1 & 0.0381 & 0.0381 & 0.0381 & 0.0381 & 0.0381 & 0.0381 & 0.0381 & 0.0381 & 0.0381 \\
\hline $\begin{array}{l}\text { Brass } \\
\text { thickness } \\
(\mathrm{cm})\end{array}$ & 0.0127 & 0.0254 & 0.0381 & 0.0813 & 0.0127 & 0.0127 & 0.0254 & 0.0381 & 0.0813 & 0.0127 & 0.0127 & 0.0127 & 0.0127 \\
\hline
\end{tabular}

"For all samples: Young's modulus of PZT, $62.5 \mathrm{GPa}$; Young's modulus of brass, $110 \mathrm{GPa}$; and $d_{31}$ of PZT, $-295 \times 10^{-12} \mathrm{mV}^{-1}$. D is circular disk; $\mathrm{S}$ is square plate; and $\mathrm{R}$
is rectangular plate.
is rectangular plate.

(a)

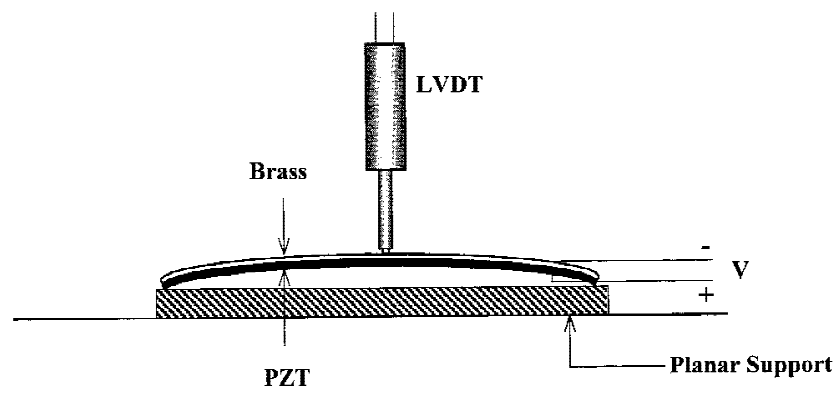

(b)

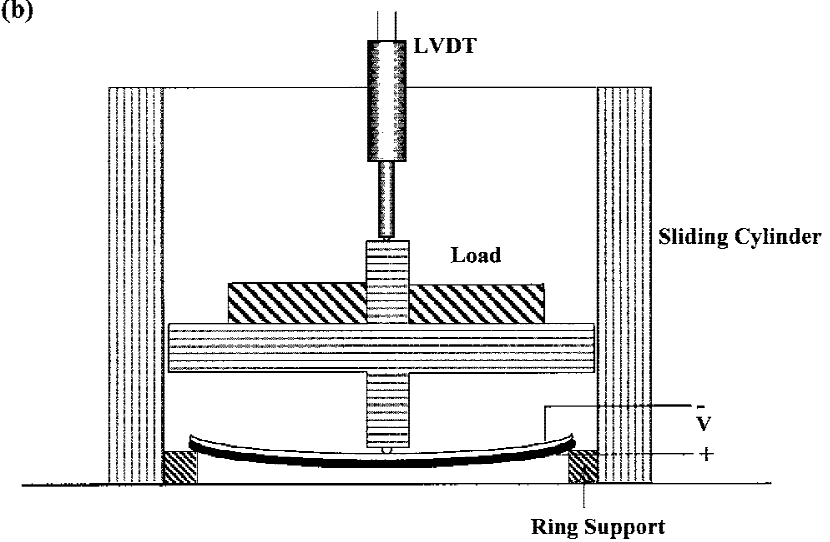

Fig. 3. Experimental setup for the measurement of displacement under (a) planar and (b) ring supports.

schematically shown in Figs. 3(a) and (b), respectively. As shown in Fig. 3(a), without a load, the displacement measurements were done with the displacement transducer placed directly on top of the specimen. For measuring the displacements under the loading conditions, a load cell consisting of an outer cylinder and a weight holder that was shaped similar to a dreidel was used. The displacement was measured with the top of the weight holder against the displacement transducer. A load was applied to a unimorph by placing ring-shaped weights on the weight holder that had a ball tip (made of case iron) $\sim 1 \mathrm{~mm}$ in diameter touching the center of the unimorph. The outer cylinder kept the weight holder from wobbling, while the inner surface of the cylinder was smooth, such that the weight holder could move up and down freely during the displacement measurement.

The resonance frequencies of the unimorphs were measured with an impedance analyzer (Model HP4192, Hewlett-Packard, Palo Alto, CA) in the frequency range of $100 \mathrm{~Hz}$ to $10 \mathrm{MHz}$. To avoid the clamping effect to the resoance frequency, measurements were done under free-standing conditions.

\section{Experimental Results and Discussion}

As an example, the displacement of a PZT-brass unimorph (D3) as a function of the voltage applied across the thickness of the unimorph is shown in Fig. 4. The displacement generally increases with increasing applied electric field, with some hysteresis. The displacement obtained when the applied voltage is decreased from 600 to $0 \mathrm{~V}$ is somewhat larger than that obtained when the voltage is increased from 0 to $600 \mathrm{~V}$. A similar phenomenon has been shown in Moonie transducers by Dogan et al..$^{13}$ and in gradient transducers by Wu et al. ${ }^{14}$ The behavior shown in Fig. 4 is similar to the strain versus field behavior of PZT, except for the larger displacements of the unimorph. The hysteretic behavior of the unimorph is attributed to the switching of polarization domains in PZT when an electric field is increased or decreased. To facilitate domain switching, a certain electric field is required to overcome the domain wall inertia. This is probably the reason for the small displacements at low fields $(0-100 \mathrm{~V})$ and where fields are reversed $(700-800$ $\mathrm{V})$. Here, the dc electric field is reversed by switching the knob on the power supply. Furthermore, because the resolution of the displacement measurement is $1 \mu \mathrm{m}$, the small change in the displacement when the field is increased from 0 to $100 \mathrm{~V}$ or decreased from 800 to $700 \mathrm{~V}$ cannot be detected. When the brass is on the top, the displacement of the unimorph is upward when the applied electric field is parallel to the polarization of the PZT. When the direction of the electric field is opposite to that of the polarization of the PZT, provided the field is <400 $\mathrm{V} / \mathrm{mm}$ so that no depoling of the sample occurs, the obtained displacement is similar in magnitude but reversed in direction, i.e., moving downward.

The effect of the brass/PZT thickness ratio, $t_{\mathrm{np}} / t_{\mathrm{p}}$, at a fixed PZT layer thickness is shown in Fig. 5, where the displacement, $h$, of the unimorph is plotted versus $t_{\mathrm{np}} / t_{\mathrm{p}}$. To avoid a large hysteresis effect, moderate driving voltages are used for the displacement measurement. The data points shown in Fig.

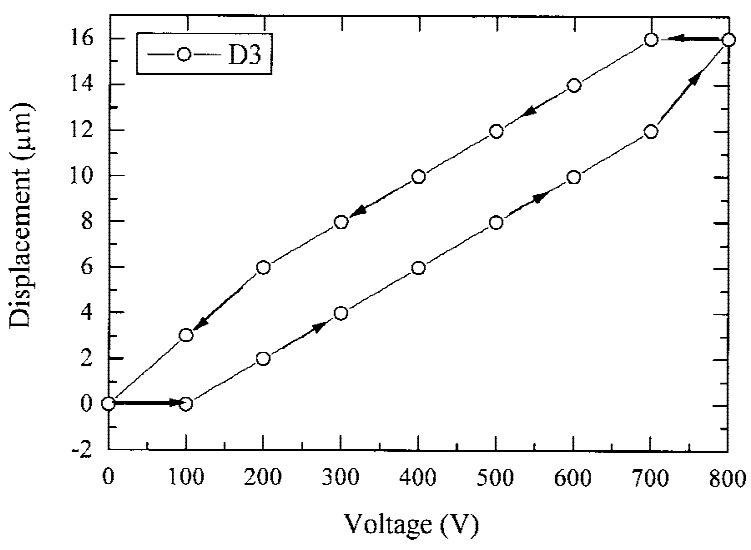

Fig. 4. Behavior of displacement of a PZT-brass disk as a function of the applied voltage. 


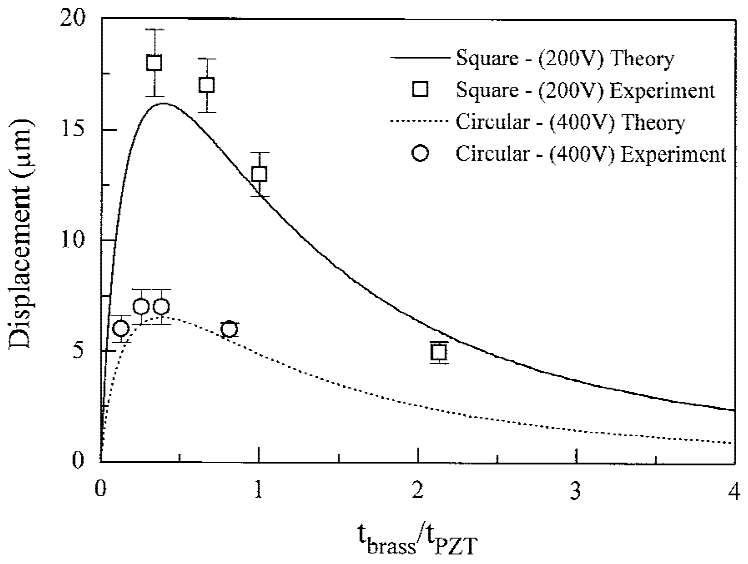

Fig. 5. Displacement of unimorph at a constant voltage as a function of the brass/PZT thickness ratios for squares and disks. Applied voltage for disks and squares are different.

5 are the average of four measurements on each sample, where the applied voltages are 200 and $400 \mathrm{~V}$ for the square and disk unimorphs, respectively. Also plotted are the calculated curves for the square unimorphs (solid line), using Eq. (12), and for the disk unimorphs (dashed line), using Eq. (15). The calculated curves are obtained using $E_{\mathrm{p}}=62.6 \mathrm{GPa}, E_{\mathrm{np}}=110$ $\mathrm{GPa}$, and $d_{31}=-295 \times 10^{-12} \mathrm{mV}^{-1}$, as specified in Table I. The calculated curves are in close agreement with the experimental results. Equation (12) is obtained assuming that the square unimorph is supported at the four corners, and Eq. (15) is obtained assuming that the disk unimorph is supported at the rim. The close agreement between the calculated curves and the experimental results suggests that these boundary conditions are quite reasonable for the present experimental setup. For both the square and the disk unimorphs, the highest displacement occurs at the center of the unimorph. However, the displacement of the circular unimorph vanishes at every point of the rim, whereas that of the square unimorph vanishes only at the four corners. Consequently, a square unimorph is better than a circular unimorph for producing large displacements. Comparing Eqs. (12) and (15) shows that, for a given set of $t_{\mathrm{p}}$ and $t_{\mathrm{np}}$ and for a fixed area, a square unimorph produces a displacement $\pi / 2$ times as high as does a circular unimorph.

Meanwhile, the calculated curves for both the square and the disk unimorphs exhibit a maximum at $t_{\mathrm{np}} / t_{\mathrm{p}} \approx 0.38$. This can be explained as follows. Denoting $\alpha=t_{\mathrm{np}} / t_{\mathrm{p}}$ and $a=$ $E_{\mathrm{np}} / E_{\mathrm{p}}$, the maximum in $h$ occurs at $\partial h / \partial \alpha=0$. For both the disk and the square unimorphs,

$$
\frac{\partial h}{\partial \alpha}=1+2 \alpha-2 a \alpha^{2}-8 a \alpha^{3}-\left(4 a+3 a^{2}\right) \alpha^{4}-3 a^{2} \alpha^{5}=0
$$

Thus, with $E_{\mathrm{p}}=62.6 \mathrm{GPa}$ and $E_{\mathrm{np}}=110 \mathrm{GPa}, \partial h / \partial \alpha=0$ occurs at $\alpha=0.38$ for both the square and the disk unimorphs, which agrees very well with the experimental results. The disk unimorphs show a maximum in displacement at $t_{\mathrm{np}} / t_{\mathrm{p}} \approx 0.38$. Even though the square unimorphs lack data points at $t_{\mathrm{np}} / t_{\mathrm{p}}<$ 0.38 , the displacement is also the highest at $t_{\mathrm{np}} / t_{\mathrm{p}} \approx 0.38$.

Equation (24) applies to the case of constant PZT thickness. The results for the case of constant total thickness has been published earlier ${ }^{9}$ and is cited here for the purpose of comparison. The condition for optimal displacement of a unimorph with a fixed total thickness, $t$, is

$$
\frac{t_{\mathrm{np}}}{t_{\mathrm{p}}}=\sqrt{\frac{E_{\mathrm{p}}}{E_{\mathrm{np}}}}
$$

Therefore, if the total thickness of the unimorph is fixed, the optimal brass/PZT thickness ratio $t_{\mathrm{np}} / t_{\mathrm{p}}=0.75$. The result for

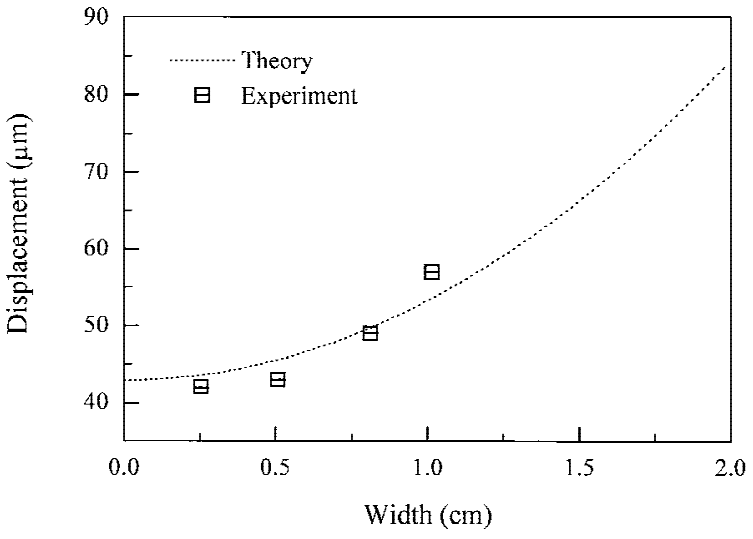

Fig. 6. Displacement of rectangular unimorph with fixed length as a function of the width of the rectangles (length $=2.032 \mathrm{~cm}, t_{\mathrm{brass}}=$ $0.0127 \mathrm{~cm}$, and $t_{\mathrm{PZT}}=0.0381 \mathrm{~cm}$ at $200 \mathrm{~V}$ ).

a fixed total thickness is very different from that of a fixed PZT thickness. Also, the optimal thickness ratio is relatively insensitive to the uncertainty in Young's modulus, $E_{\mathrm{p}}$. For example, an error of $10 \%$ in $E_{\mathrm{p}}$ results in a $5 \%$ change in the optimal thickness ratio, according to Eq. (25), and a 3\% change according to Eq. (24). In principle, the Young's modulus of PZT may change because of domain switching when an electric field is applied. However, because of the insensitivity of the thickness ratio to Young's modulus, we expect the effect of domain switching on the thickness ratio is small.

According to Eq. (11), the maximum displacement at the center of a rectangular unimorph increases if the width of the rectangular unimorph is increased while its length is held fixed. This trend is illustrated in Fig. 6, where the maximum displacement at the center of a rectangular unimorph is plotted versus the width of the rectangular unimorph and the length of the rectangular unimorphs is fixed at $2.03 \mathrm{~cm}$. The rectangular unimorphs have $t_{\mathrm{np}}=0.0127 \mathrm{~cm}$ and $t_{\mathrm{p}}=0.0381 \mathrm{~cm}$. As the width of the rectangular unimorph increases from 0.254 to 1.01 $\mathrm{cm}$, the displacement of the unimorph increases in close agreement with the calculated curve (dashed line), using Eq. (11).

The displacements of a disk unimorph under the loadcarrying conditions also have been examined with the disk unimorph sitting on a ring (ring support) as well as on a flat surface (planar support). Figure 7 shows the maximum displacement at the center of a disk unimorph versus the load. A voltage of $200 \mathrm{~V}$ is applied across the thickness of the unimorph. In each measurement, a load is placed on the top of the unimorph, as schematically shown in Fig. 3, before the electric

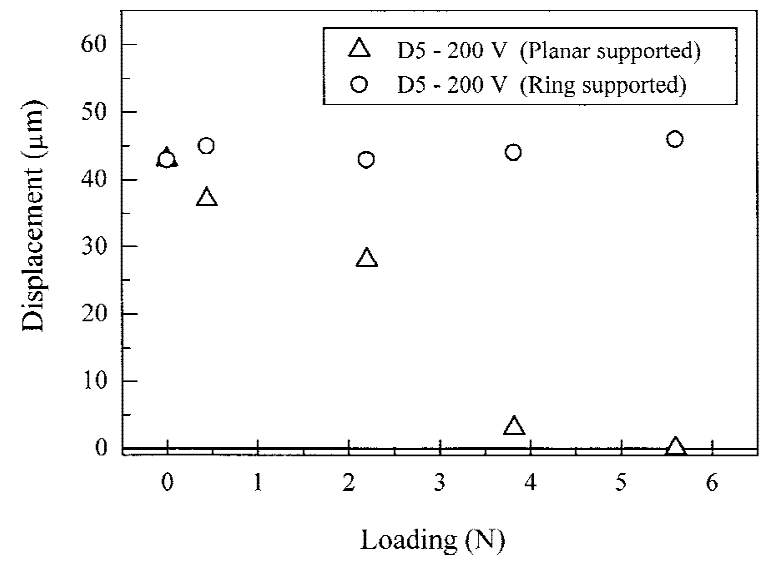

Fig. 7. Displacement behavior of unimorph as a function of loading for planar and ring supports. 

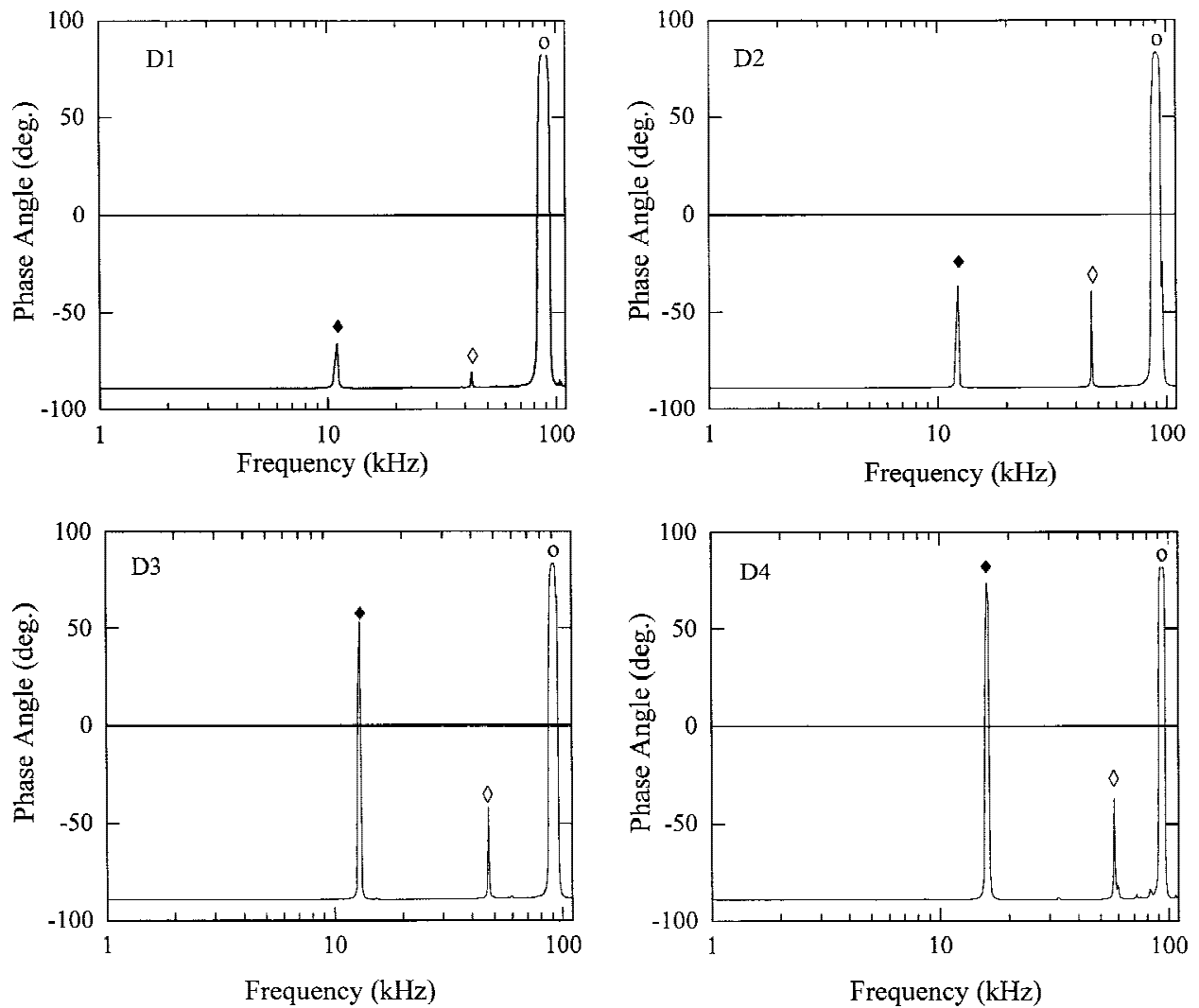

Fig. 8. Phase angles of disk unimorph as a function of frequency for disks of different brass/PZT thickness ratios. From samples D1-D4, the brass thickness increases and PZT thickness is held constant. ( $\diamond)$ first bending mode and $(\diamond)$ second bending mode.)

field is applied. With a planar support, the maximum displacement decreases with an increasing load. However, with a ring support, the displacement is relatively independent of the load. The difference between the displacements measured with a ring support and that with a planar support can be explained as follows.

Under the loading conditions, the displacement is obtained by measuring the difference in the positions at the top of the load before and after the electric field is applied. In the case of ring support, the unimorph is able to respond to the load by moving downward before the electric field is applied, and the zero point of the displacement corresponds to the position of the top layer at zero field and under a load. Therefore, with a ring support, the measured displacement is due only to the applied voltage. Furthermore, because the applied voltage is fixed at $200 \mathrm{~V}$, the measured displacement is independent of the load. On the other hand, with a planar support, the unimorph cannot move downward in response to the load; therefore, the zero point of the displacement corresponds to the position of the top layer at zero field and zero load. When the electric field is applied, part of the lateral stress generated by the applied field is compensated by that produced by the load. Consequently, the displacement decreases with increasing load. The effect of a load with a planar support is qualitatively explained in the theory section by considering a beam with a concentrated load $F$ at the center. The displacement of the unimorph is simply reduced with a load by an amount equal to the term involving the load $F$ in Eq. (18). Thus, the displacement decreases linearly with increasing load, which qualitatively agrees with the experimental results shown in Fig. 7.

The dynamic response and the resonance frequencies of the the unimorph have been measured using an impedance analyzer. Figure 8 shows the phase angles of disk samples as a function of frequency. There are many resonance peaks that correspond to various vibrational modes. The first resonance peak corresponds to the lowest bending mode. A derivation for the bending-mode resonance frequencies is given in the Theory section. The bending resonance frequency is proportional to $\left(D^{\prime} / m\right)^{1 / 2} L^{-2}$, as shown in Eq. (22). $D^{\prime}$ is the bending modulus per unit length, which depends on the modulus of each layer, the thickness of each layer, the mass per unit area, and the width $(L)$ of the unimorph, as shown in Eq. (23). The normalized bending resonance frequency, $f L^{2} /\left(D^{\prime} / m\right)^{1 / 2}$ as a function of the brass thickness is shown in Fig. 9. Similar results of phase angles as a function of frequency for square samples are shown in Fig. 10, and the normalized resonance frequencies as a function of the brass thickness are shown in Fig. 11. The normalization of the resonance frequency has been included to compare the experimental results with the theoretical prediction. According to Eq. (22), dividing the frequency by the factor $\left(D^{\prime} / m\right)^{1 / 2} L^{-2}$, the normalized frequency, $f L^{2} /\left(D^{\prime} / m\right)^{1 / 2}$, is

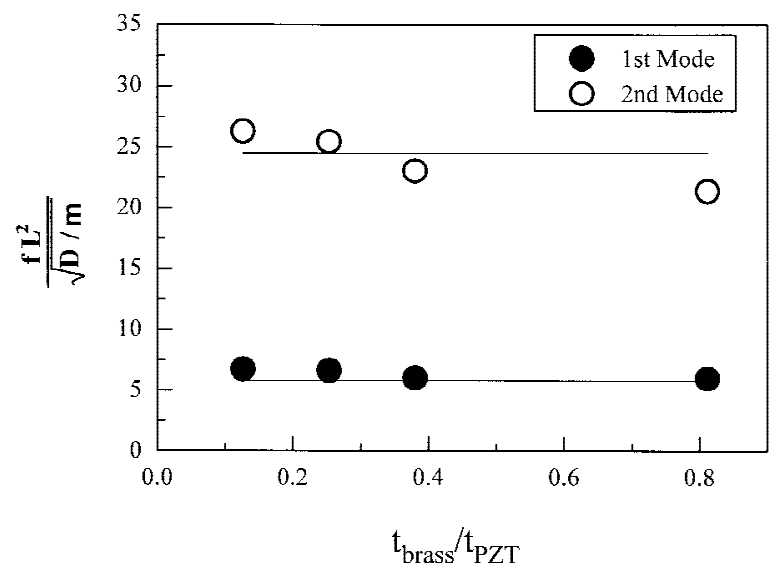

Fig. 9. Normalized resonance frequency as a function of brass/PZT thickness ratio for disk unimorph. Lines are theoretical predictions. 

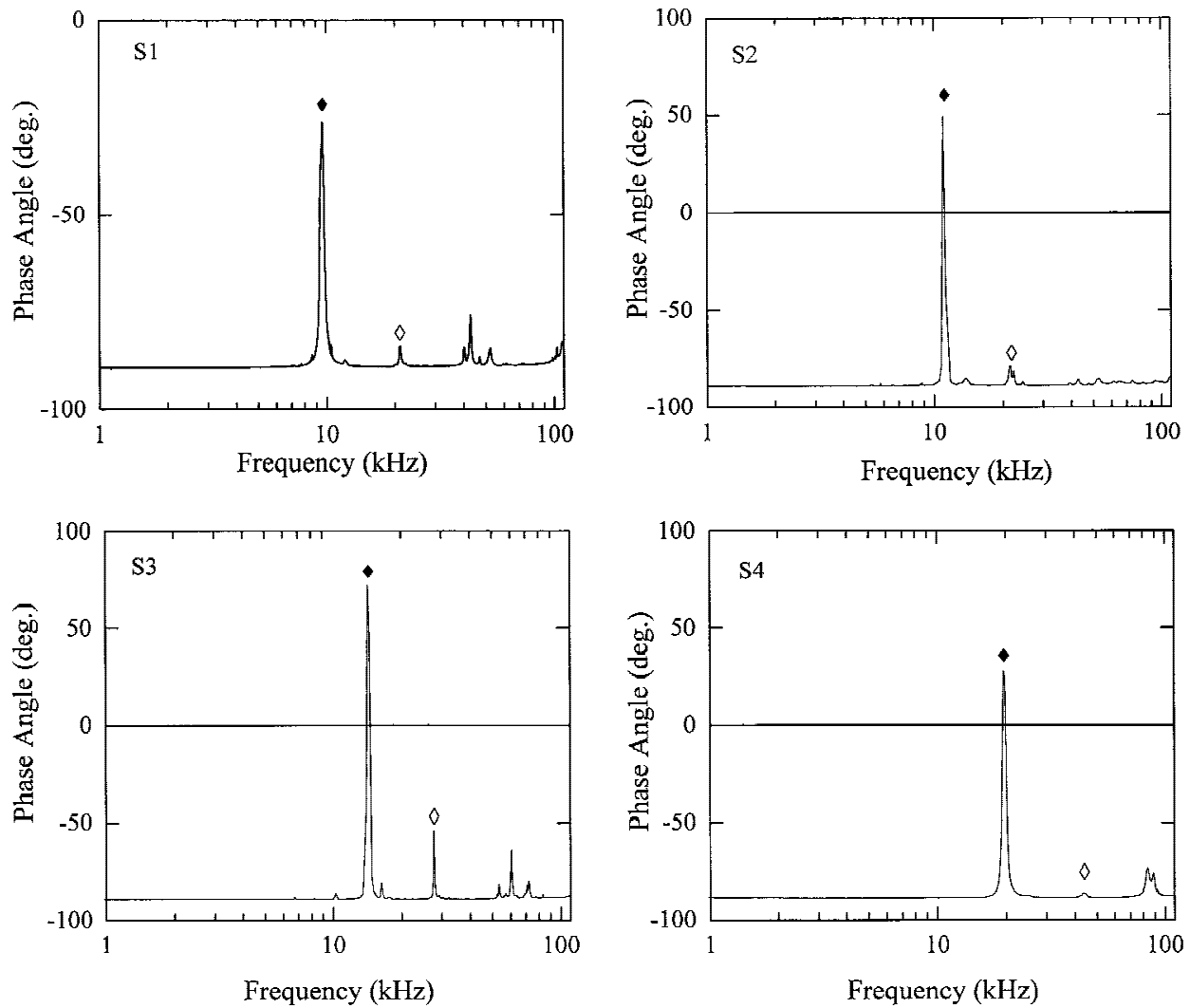

Fig. 10. Phase angles of disk unimorph as a function of frequency for square unimorph of different brass/PZT thickness ratios. From samples S1-S4, the brass thickness increases and PZT thickness is held constant. $((\diamond)$ first bending mode, $(\diamond)$ second bending mode, and (o) fundamental radial mode.)

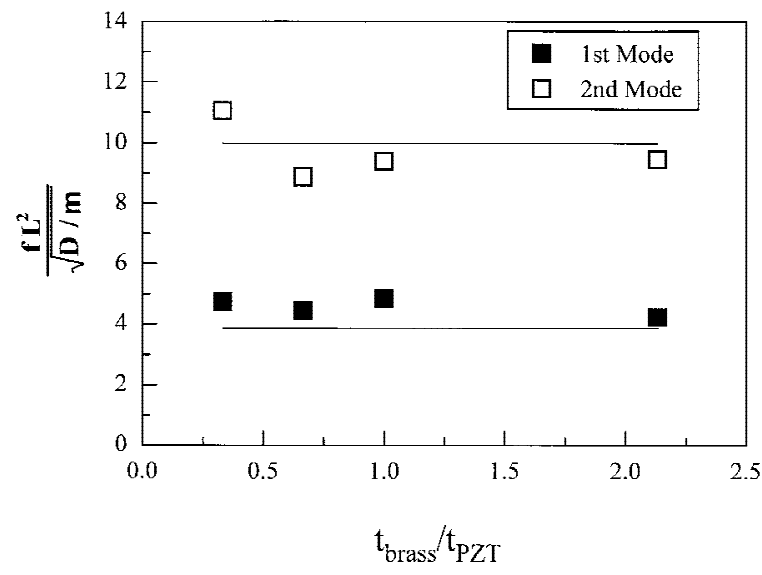

Fig. 11. Normalized resonance frequency as a function of brass/PZT thickness ratio for square unimorphs. Lines are theoretical predictions.

a constant that equals $\lambda^{2} / 2 \pi$, where $\lambda$ is the eigenvalue of Eq. (19). For the free-standing boundary condition that we have used in the experiment, the values of $\lambda^{2} / 2 \pi$ corresponding to the first two bending modes are 5.78 and 24.54 for disk samples and 3.86 and 10.14 for square samples. ${ }^{12}$ Figures 9 and 11 show that the normalized resonance frequency is independent of the brass thickness. The horizontal lines are the calculated $\lambda^{2} / 2 \pi$ values for the first and second modes, respectively. There is a good agreement between the theoretical prediction and the experimental results.

\section{Conclusion}

We have shown that the electromechanical behavior of piezoelectric-nonpiezoelectric unimorphs varies with the thickness ratios of the two layers. The axial displacement of the unimorph under an applied electric field shows a maximum at a specific thickness ratio. The experimental results agree well with the theory of plates. Square samples show higher displacement than circular samples, in agreement with the theoretical prediction. Loading does not change the displacement of a unimorph if it is placed on a ring support. On the other hand, loading decreases the displacement if the unimorph is placed on a planar support. Furthermore, for a given PZT thickness, the resonance frequency of the unimorph varies with the brass/PZT thickness ratio. The resonance frequency behavior can be understood by the mechanical vibrations of plates.

Acknowledgments: Supported by the Army Research Office Multidisciplinary University Research Initiative (ARO/MURI) under Grant No. DAAH04-95-1-0102. We thank W. L. Worrell of University of Pennsylvania for letting us use his impedance analyzer.

\section{References}

${ }^{1}$ Y. Sugawara, K. Onitsuka, S. Yoshikawa, Q. C. Xu, R. E. Newnham, and K. Uchino, "Metal-Ceramic Composite Actuators," J. Am. Ceram. Soc., 75 [4] 996-98 (1992).

${ }^{2}$ G. H. Haertling, "Rainbow Ceramics-A New Type of Ultra-High-Displacement Actuator," Am. Ceram. Soc. Bull., 73 [1] 93-96 (1994).

${ }^{3}$ C. Elissalde, L. E. Cross, and C. A. Randall, "Structural-Property Relations in a Reduced and Internally Biased Oxide Wafer (RAINBOW) Actuator Material," J. Am. Ceram. Soc., 79 [8] 2041-48 (1996).

${ }^{4}$ C. Elissalde and L. E. Cross, "Dynamic Characteristics of Rainbow Ceramics," J. Am. Ceram. Soc., 78 [8] 2233-36 (1995).

${ }^{5}$ B. W. Barron, G. Li, and G. H. Haertling, "Electromechanical Properties of PLZT-Based Cerambow Actuators," Abstract Book, 99th Annual Meeting of The American Ceramic Society; p. 190, Paper No. SXVII-015-97, 1997.

${ }^{6}$ (a)R. F. Hellbaum, R. G. Bryant, and R. L. Fox, "Thin Layer Composite Unimorph Ferroelectric Driver and Sensor," U.S. Pat. No. 5632 841, May 27, 1997. (b)R. G. Bryant, "Process for Preparing a Tough, Soluble, Aromatic, Thermoplastic Copolyimide," U.S. Pat. No. 5639 850, June 17, 1997.

${ }^{7}$ M. R. Steel, F. Harrison, and P. G. Harper, "The Piezoelectric Bimorph: An 
Experimental and Theoretical Study of Its Quasistatic Response," J. Phys. D Appl. Phys., 11, 979-89 (1978).

${ }^{8}$ J. G. Smits, S. I. Dalke, and T. K. Cooney, "The Constitutent Equations of Piezoelectric Bimorphs," Sens. Actuators A, 28, 41-61 (1991).

${ }^{9}$ W. Y. Shih, W.-H. Shih, and I. A. Aksay, "Scaling Analysis for the Axial Displacement and Pressure of Flextensional Transducers," J. Am. Ceram. Soc., 80 [5] 1073-78 (1997).

${ }^{10} \mathrm{G}$. Li. E. Furman, and G. H. Haertling "Stress-Enhanced Displacements in PLZT Rainbow Actuators," J. Am. Ceram. Soc., 80 [6] 1382-88 (1997).

${ }^{11}$ K. Onitsuka, A. Dogan, Q. Xu, S. Yoshikawa, and R. E. Newnham, "De- sign Optimization for Metal-Ceramic Composite Actuator (Moonie)," Ferroelectrics, 156, 37-42 (1994).

${ }^{12}$ A. W. Leissa, "Vibration of Plates," NASA SP-160, Scientific and Technical Information Division, Washington, DC, 1969

${ }^{13}$ A. Dogan, Q. Xu, K. Onitsuka, S. Yoshikawa, K. Uchino, and R. E. Newnham, "High-Displacement Ceramic-Metal Composite Actuators (Moonies)," Ferroelectrics, 156, 1-6 (1994).

${ }^{14} \mathrm{C}$. M. Wu, M. Kahn, and W. Moy, "Piezoelectric Ceramics with Functional Gradients: A New Application in Material Design," J. Am. Ceram Soc., 79 [3] 809-12 (1996). 